\title{
Segregação Escolar e Desigualdades Educacionais no Início da Escolarização no Brasil
}

\author{
School Segregation and Education Inequalities at the Start of \\ Schooling in Brazil
}

\author{
Tiago Lisboa Bartholo* \\ Mariane Campelo Koslinski \\ Felipe Macedo de Andrade \\ Daniel Lopes de Castro
}

Universidade Federal do Rio de Janeiro, Brasil

\begin{abstract}
O artigo descreve padrões de segregação escolar no início do processo formal de escolarização na cidade do Rio de Janeiro, Brasil, e estima o efeito da segregação escolar (efeito par ou efeito mistura/compositional effect) no aprendizado das crianças nos dois primeiros anos na escola. Este é um fenômeno importante para analisar o processo de distribuição de oportunidades educacionais nas redes públicas e privadas de ensino. O conceito de segregação escolar utilizado neste artigo se refere à distribuição desigual de alunos que partilham de uma característica específica em um agrupamento de escolas. O estudo utiliza o Índice de Segregação (Segregation Index) e considera duas características dos alunos: 1) cor/raça; 2 ) escolaridade dos pais. O efeito da composição social foi estimado com dados de um estudo longitudinal e analisa separadamente o aprendizado no primeiro e segundo ano na escola. Os resultados preliminares sugerem que os padrões de segregação escolar observados na pré-escola são semelhantes aos descritos no primeiro ano do ensino fundamental. Não há, portanto, grande impacto nos padrões de segregação na transição entre as etapas de ensino. Os modelos para estimar o efeito da composição social das escolas (compositional effect) no aprendizado dos alunos sugerem que há efeito, com resultados mais consistentes para a medida de linguagem.
\end{abstract}

Palavras-chave: Segregação escolar; Desigualdades educacionais; Efeito par; Estudo Longitudinal; Pré-escola.

The paper describes patterns of school segregation at the start of the formal schooling in the city of Rio de Janeiro, Brazil, and estimates the compositional effect (school mix-effect) on children's learning in the first two years at school. This is an important phenomenon to analyze the process of distributing educational opportunities in public and private schools. The concept of school segregation used in this paper refers to the unequal distribution of students who share specific characteristics within a group of public schools. The study uses the Segregation Index for two characteristics of disadvantaged: 1) color/race; 2) parents' education. The effect of social composition was estimated with data from a longitudinal study and analyzed separately the compositional effect for the first and second years at school. Preliminary results suggest that the patterns of school segregation observed in preschool are similar to those described in the first year of elementary school. There is, therefore, no major impact on segregation patterns in the transition between teaching stages. The models for estimating the effect of the compositional effect on student learning suggest that there is an effect, with more consistent results for the measurement of language.

Keywords: School segregation; School inequality; Compositional effect; Longitudinal Study; Preschool.

\section{*Contacto: tiagobartholo@ufrj.br}

ISSN: $1696-4713$

www.rinace.net/reice/

revistas.uam.es/reice
Recibido: $\quad 15$ de mayo 2020

$1^{\text {a }}$ Evaluación: 25 de junio 2020



Aceptado: 25 de agosto 2020 


\section{Introdução}

$\mathrm{O}$ artigo descreve padrões de segregação escolar no início do processo formal de escolarização na cidade do Rio de Janeiro, Brasil, e estima o efeito potencial da segregação escolar (efeito par ou efeito mistura/compositional effect) no aprendizado das crianças nos dois primeiros anos na escola. ${ }^{1}$ Estudos anteriores realizados no Brasil, evidenciaram que a segregação escolar é um fenômeno relevante para pensar a distribuição de oportunidades educacionais nas redes públicas e privadas de ensino (Bartholo e Costa, 2018, 2016; Mendes, 2017).

O tema não é novo e já foi investigado utilizando dados secundários na cidade do Rio de Janeiro e em outras cidades do Brasil (Bartholo e Costa, 2016; Bruel e Bartholo, 2012; Carvalho Filho, 2016). Há, entretanto, duas dimensões inéditas nesse trabalho: 1) o uso de indicadores de segregação escolar para descrever a distribuição de alunos na pré-escola (4 e 5 anos), período obrigatório do processo de escolarização desde 2009; 2) a análise do efeito da segregação escolar no aprendizado dos alunos nos primeiros dois anos na escola, utilizando com dados com características longitudinais.

Nesse artigo, os autores adotam uma definição específica para o fenômeno de segregação escolar. Trata-se de uma distribuição desigual de estudantes que compartilham uma determinada característica específica em um agrupamento de escolas. O fenômeno foi calculado com base no Índice de Segregação (Segregation Index, GS) (Gorard, Taylor e Fitz, 2003) e seu uso se justifica, em grande parte, por ser um índice apropriado para análises longitudinais (Murillo, 2016). O conceito de segregação não deve ser interpretado como um sinônimo de discriminação ou injustiça. De fato, diante da estratégia adotada para mensurar o fenômeno, a segregação é quase inevitável. O debate sobre segregação escolar está intimamente ligado à temática da distribuição das oportunidades educacionais e, de forma mais ampla, à justiça social (Rawls, 1971). Trata-se de um fenômeno universal que está associado com a segregação residencial, escolha parental e políticas educacionais. Outros fatores relacionados com a desigualdade social, isolamento social, econômico e cultural ajudam a explicar parte da variação observada no fenômeno (Harris, 2012).

É possível dividir os estudos sobre segregação escolar em dois grupos. A primeira busca descrever os padrões de segregação escolar e identificar fatores associados ao fenômeno. Estudos no campo da educação focam em especial na formulação de políticas públicas que podem impactar a alocação dos alunos nas escolas. Um exemplo são as políticas da escolha escolar (school choice) que já foram objeto de análises em diversos países com uma discussão intensa sobre seus efeitos nos padrões de segregação escolar em diferentes países (Goldhaber, 1995; Gorard, Taylor e Fitz, 2003; Santos e Elacqa, 2016).

O segundo grupo analisa os impactos da segregação na trajetória e/ou resultados educacionais dos alunos. Estudos realizados em diferentes países sugerem efeitos distintos. Por um lado, é possível formular o argumento que agrupar alunos com características semelhantes, por exemplo, estudantes com dificuldades de aprendizado, pode ser eficaz quando se pretende implementar políticas direcionadas para ajudar tais

\footnotetext{
${ }^{1} \mathrm{O}$ estudo contou com financiamento do Banco Interamericano de Desenvolvimento (IADB), Fundação de Amparo a Pesquisado do Estado do Rio de Janeiro (FAPERJ), Conselho Nacional de Desenvolvimento Científico e Tecnológico (CNPq) e Instituto Alfa e Beto (IAB).
} 
grupos. Por outro lado, há evidencias que sugerem que agrupar alunos com desvantagem potencial (por exemplo, alunos pobres ou com dificuldades de aprendizado) pode influenciar a forma como são tratados na escola, suas aspirações educacionais ou a qualidade do ensino. Sistemas educacionais mais segregados ou com políticas que intencionalmente agrupam alunos com características semelhantes possuem maior associação entre o nível socioeconômico das famílias e os níveis de aprendizado dos alunos. (Haahr et al., 2005; Jenks et al., 1972; Quiroz, Dari e Cervini, 2020).

O presente artigo está dividido em cinco partes incluindo a introdução. A segunda parte faz uma revisão dos principais achados produzidos no Brasil sobre os padrões de segregação escolar nas redes pública e privada e também dos seus efeitos nos resultados escolares dos alunos. A terceira parte descreve as bases de dados utilizadas nas análises e os modelos de análise propostos. A quarta parte apresenta os principais achados do estudo e realiza um diálogo com estudos prévios realizados no Brasil e outros países da américa latina. Por fim, a quinta parte apresenta conclusões preliminares e sugere lacunas e análises futuras.

\section{Segregação escolar no Brasil: O que já sabemos?}

Estudos sobre segregação escolar que descrevem os padrões de segregação e identificam fatores que explicam o fenômeno no Brasil têm focado suas análises em estudantes da rede pública de ensino (Bartholo, 2013; Bruel e Bartholo, 2012; Carvalho, 2015; Érnica e Batista, 2012; Mendes, 2017). Há um único estudo que conhecemos que utilizou dados populacionais e mensurou a segregação escolar incorporando dados das redes público e privada em quatro capitais no Brasil, a saber, Rio de Janeiro, São Paulo, Belo Horizonte e Curitiba (Bartholo e Costa, 2018).

Os elementos estudados sobre o fenômeno da segregação escolar no Brasil podem ser agrupados nas seguintes categorias: a) Estratificação entre as redes pública e privada; b) Regras de matrícula para alocação de alunos na rede pública; c) Estratégias familiares para a escolha escolar; d) Padrões de segregação residencial e sua relação com a segregação escolar; e) Movimentação de alunos entre escolas; f) Alocação nos turnos escolares e; g) efeito da composição social das escolas no aprendizado dos alunos. Vamos falar de forma mais detalhada sobre cada um dos sete pontos.

No Brasil, a proporção de estudantes matriculados na rede privada de ensino é substancialmente maior do que o observado nos EUA, e na grande maioria dos países europeus. A proporção de alunos no ensino fundamental (idade entre 6 e 14 anos) matriculados na rede privada aumentou, entre 2010 e 2014 , de $13 \%$ para $16 \%$ aproximadamente. Entre 2015 e 2019 o número se manteve quase estável chegando a 17\%. No entanto, quando analisamos dados discriminados por diferentes cidades é possível observar grande variação. Na cidade do Rio de Janeiro, a proporção de matrículas na rede privada aumentou de 29\% em 2010, para 33\% em 2014, e praticamente ficou estável neste patamar até 2019. Já na cidade de Fortaleza, capital do Estado do Ceará, os números são ainda maiores: representavam 36\% das matrículas em 2010, 44\% em 2014, com uma leve queda, chegando a $42 \%$ em 2019.

Esse contexto cria um dilema para pesquisadores interessados no tema da segregação escolar. Ao optar por trabalhar apenas com dados da rede pública, há necessariamente uma subestimação do fenômeno da segregação escolar. Isso ocorre porque a alocação de 
famílias entre escolas públicas e privadas não é aleatória e é fortemente marcada pelo perfil socioeconômico das famílias que desejam e podem pagar mensalidades nas escolas privadas.

Bartholo e Costa (2018) realizaram estudo, utilizando dados para toda a população de alunos, mensurando o efeito da inclusão das matrículas da rede privada nos níveis de segregação medidos ao longo de 10 anos (2007 até 2016). Os dados evidenciam um incremento importante nos níveis de segregação escolar observados em quatro cidades no Brasil. Por exemplo, na cidade do Rio de Janeiro, os dados mostram um salto no cálculo do Segregation Index (GS) de 15\% para 25\% quanto incorporados os dados da rede privada para alunos negros.

Estudos sobre o impacto das regras de matrículas já foram realizados em diferentes países do mundo incluindo o Brasil. As regras de matrícula podem permitir ou não a escolha dos responsáveis sobre a escola que seu filho será matriculado. Evidências ao redor do mundo apresentam resultados mistos sobre o tema. Por exemplo, Gorard, Taylor e Fitz (2003) realizaram acompanhamento transversal ao longo de 20 anos calculando os níveis de segregação escolar no sistema inglês de ensino e sugerem que a implementação de políticas de escolha parental (School Choice) está associada com uma diminuição dos níveis de segregação escolar. No entanto, estudos realizados no contexto chileno observam aumento da segregação escolar, para além da segregação residencial, com a adoção da política de voucher. Tal situação poderia ser explicada pela baixa regulamentação do quasemercado escolar chileno, ou seja, pela interação entre escolha dos pais e as barreiras impostas por cobrança de taxas e por processos de admissão seletivos (Santos e Elacqua, 2016).

Outro grupo de estudos -difundido em diversos contextos, em especial no norteamericano- investiga a associação entre padrões de segregação residencial ou socioespacial e de segregação escolar (Denton, 1995; Frankenberg, 2013; Santos e Elacqua, 2016). No entanto, no contexto brasileiro, esta relação é tratada somente de forma indireta. Por exemplo, alguns estudos que buscaram observar a relação entre a segregação residencial e os resultados escolares sugerem, para além da influência de mecanismos de socialização na vizinhança, um efeito da segregação socioespacial sobre a oferta escolar (distribuição desigual de insumos escolares, composição do alunado e relações professor-aluno em áreas de maior concentração de pobreza) (Koslinski, Alves e Lange, 2013). Outros estudos observaram a relação entre a segregação socioespacial, a possibilidade de escolha e movimentação entre escolas públicas de maior ou menor prestígio, o que, por sua vez, teria impacto sobre a segregação escolar (Koslinski et al., 2014).

A movimentação de alunos entre escolas também é objeto de análise por pesquisadores de diferentes países. A hipótese aventada sugere que as movimentações não são aleatórias. De um lado há percepções distintas dos responsáveis sobre a qualidade das escolas e estratégias distintas para obter vaga nas escolas de melhor reputação. De outro lado, as próprias escolas podem ser agentes que maximizam a movimentação dos estudantes sugerindo a troca dos estudantes, impondo barreira para a entrada de alunos de certo perfil ou ainda via reprovação dos estudantes o que pode gerar ao longo dos anos a troca de escolas (Noreisch, 2007; West, Hind e Pennell, 2004; Zanten, 2005).

A movimentação não regulada de estudantes foi analisada por Saporito (2003), Bruel e Bartholo (2012), Bartholo (2014); Koslinski e outros (2014), e sugerem que a transferência 
de estudantes de uma escola para outra do mesmo sistema de ensino não é aleatória e pode aumentar os níveis de segregação escolar. No caso de algumas redes de ensino no Brasil, a ausência de regras claras e a falta de transparência do gestor público podem gerar procedimentos previstos na lei, como por exemplo, entrevistas informais com os responsáveis e seleção dos alunos baseada no rendimento escolar dos anos anteriores (Alves et al., 2015; Koslinski e Carvalho, 2015; Rosistolato et al., 2019).

Um último elemento estudado na cidade do Rio de Janeiro, que possivelmente está presente em outras redes de ensino em países em desenvolvimento, com população ainda jovem, é a alocação de alunos de forma não aleatória entre turnos escolares (Bartholo e Costa, 2014). Foi observado até 2014 que 90\% das escolas apresentavam dois turnos de ensino, manhã e tarde. Nesse contexto, alunos são alocados pela direção da escola em um dos turnos e frequentam a escola pela manhã ou tarde. Esse padrão de organização das escolas é fruto de um sistema com grande demanda por ensino básico e com um número limitado de prédios/infraestrutura para atender a demanda. Os pesquisadores fizeram uma análise comparando a segregação escolar considerando a alocação nos turnos e comparando com contra factual que desconsiderava os turnos. A alocação dos alunos nos turnos aumentava de forma importante os níveis de segregação no sistema sugerindo uma alocação não aleatória (Bartholo e Costa, 2014).

Há, no entanto, um outro conjunto de estudos, menor em quantidade, que buscam estimar os impactos da segregação no desempenho ou trajetória escolar dos estudantes. Esse é um tema bastante explorado em estudos internacionais e, em menor intensidade, no Brasil. Harker e Tymms (2004) fazem importante revisão sobre o tema, em especial as dificuldades metodológicas para medir o fenômeno do efeito par (compositional effect) e como interpretar seus resultados. De acordo com os autores, este seria o efeito específico de uma variável individual agregada no nível da escola sobre uma variável de resultado escolar, para além da contribuição desta variável no nível do indivíduo. Como alunos não estão distribuídos de forma aleatória entre as escolas, podemos esperar escolas com maior ou menor concentração de alunos em situação de vulnerabilidade e/ou com maior ou menor motivação para a performance/resultados na escola. No entanto, os autores argumentam que o efeito da composição não necessariamente se resume a interação entre pares e podem refletir a interação entre a composição do alunado e ação do professor, clima escolar, envolvimento dos pais, comprometimento dos professores entre outros. Isto é, alunos reagem às estruturas/processos escolares e a seus pares e as escolas, por sua vez, reagem à composição do alunado.

No Brasil destacamos os estudos de Marino (2014), Castro (2016), Bartholo e Costa (2016) que utilizaram dados com características longitudinais para estimar o efeito par na cidade do Rio de Janeiro com coortes distintas e idades que também variam ao longo do ensino fundamental. Os resultados são mistos, mas apontam, na média, para a existência de efeito par considerando variáveis chave como pobreza e cor/raça. Os estudos têm como principal limitação o uso de medidas oriundas de avaliação externa que apresentam grande erro de mensuração no nível do indivíduo e elevado percentual de dados faltantes.

Os resultados dos estudos realizados no contexto brasileiro descritos acima têm relação com a descrição do fenômeno da segregação escolar e tentativas de identificar fatores associados à variação observada. No entanto, os dados utilizados são referentes ao ensino fundamental (crianças entre 6 e 14 anos). As análises apresentadas no presente estudo tratam de uma etapa de escolarização distinta, a pré-escola (crianças entre 4 e 5 anos). Esta 
etapa tornou-se parte obrigatória do sistema educacional brasileiro desde 2009 e a universalização do acesso a pré-escola estava previsto para 2016, de acordo com o atual Plano Nacional de Educação (Lei 13.005/2014).

\section{Desenho e métodos}

As análises realizadas para o presente estudo estão divididas em duas partes: a primeira buscou observar padrões de segregação escolar na pré-escola e no primeiro ano do ensino fundamental de escolas públicas da rede municipal do Rio de Janeiro. A segunda parte teve como objetivo compreender o efeito da composição do alunado das escolas da mesma rede de ensino sobre aprendizagem durante a pré-escola. Para tanto, as duas partes utilizaram bases de dados e modelos de análises distintos.

Para identificar os padrões de segregação escolar, utilizamos bases de dados da Sistema de Gestão Acadêmica (SGA) da rede pública municipal de educação do Rio de Janeiro. As bases de dados do referido sistema permitem identificar em que escola os alunos estão matriculados, bem como algumas características sociodemográficas tais como sexo, cor, acesso a programas de transferência de renda e escolaridade dos pais. A organização das bases permite o cálculo da segregação escolar para o período da pré-escola entre os anos de 2006 e 2013. Foram realizados o cálculo de segregação escolar para dois grupos de alunos: a) estudantes na pré-escola ( 4 e 5 anos) e; b) estudantes no primeiro ano do ensino fundamental (6/7 anos). A ideia do desenho é comparar os padrões de segregação observados na pré-escola com os padrões no $1^{\circ}$ ano do ensino fundamental, observando similaridades e diferenças nessa transição.

A té o presente momento, não é possível calcular os indicadores de segregação para os anos subsequentes para os alunos matriculados na pré-escola em função de um limite na base de dados utilizada. Os autores têm intenção em atualizar os indicadores de segregação em estudo futuro. $\mathrm{O}$ cálculo da segregação escolar para os alunos matriculados no ensino fundamental já foi realizado até o ano de 2016 (Bartholo e Costa, 2018). Para o cálculo da segregação escolar utilizamos o Índice de Segregação (GS) (Gorard, Taylor e Fitz, 2003), um indicador que apresenta o que Gorard e Taylor (2002) denominaram como uma invariância composicional robusta (strong compositionally invariant), em comparação com outro indicador muito utilizado em pesquisas educacionais, o Índice de Dissimilaridade. (DUNCAN, 1955). Para além desse elemento, em uma comparação aprofundada entre o Índice de Dissimilaridade de Duncan, o Índice de Segregação de Gorard e o Índice da Raiz Quadrada (Hutchens, 2004), Murillo (2016) argumenta que o GS possibilita comparar a segregação escolar para diferentes grupos, expressando-se, ainda, como um indicador destacado entre os três para análise longitudinal da evolução de segregação de um sistema, característica de destaque do presente trabalho.

O GS é representando pela seguinte equação (GS):

$$
\mathrm{GS}=0.5 *\left\{\sum|\mathrm{Fi} / \mathrm{F}-\mathrm{Ti} / \mathrm{T}|\right\}
$$

Onde:

Fi é o número de alunos em desvantagem potencial na escola "i”, e "i” varia de 1 até o número total de escolas no universo em foco;

F é o número total de alunos em desvantagem potencial do mesmo conjunto de escolas; 
Ti é o número de alunos na escola "i”, e "i” varia de 1 até o total de escolas no universo em foco;

T é o total de alunos nas escolas do mesmo conjunto de escolas (Gorard, Taylor y Fitz, 2003).

O Segregation Index (GS) foi calculado considerando duas características de desvantagem potencial: a) educação dos pais e; b) cor/raça. Educação dos pais foi codificada com dois pontos de corte distintos. O primeiro considera os pais que não completaram o ensino fundamental (EducEF) como característica de desvantagem potencial e o segundo considera os pais que não completaram o ensino médio (EducEM). Educação dos pais é provavelmente um dos melhores preditores de trajetória escolar de uma criança, exceto uma medida de proficiência direta do estudante (Gorard e See, 2013). A variável cor/raça foi codificada como característica de desvantagem potencial alunos pretos ${ }^{2}$. No Brasil, diversos estudos no campo da educação, economia e sociologia utilizam a cor/raça para compreender desigualdades sociais associadas não somente ao tópico das oportunidades educacionais, como também de renda, acesso ao mercado de trabalho, exposição à violência, etc.

No entanto, a variável cor nas bases utilizadas apresenta algumas limitações. Em primeiro lugar, os dados faltantes de cor para crianças matriculadas na pré-escola são mais elevados do que para o ensino fundamental, representando $17,4 \%$ e $4 \%$ dos casos, respectivamente. Além disso, a utilização mais recorrente da variável cor no contexto brasileiro é a auto declaração de cor, o que representaria limitações dada a faixa etária das crianças/alunos foco presente estudo ${ }^{3}$. Os registros das escolas, a princípio, são provenientes de informações obtidas pelos pais/responsáveis das crianças, mas em alguns casos os funcionários da secretaria da escola preenchem as informações no sistema a partir de suas percepções (hetero-atribuição), que não necessariamente estariam de acordo com a percepção dos pais.

A segunda parte das análises, que busca identificar o efeito da composição do alunado sobre a aprendizagem durante os dois anos da pré-escola, utilizou bases de um estudo longitudinal que coletou dados durante março de 2017 e dezembro de 2018 com crianças matriculadas na rede municipal do Rio de Janeiro. A pesquisa selecionou uma amostra probabilística aleatória de 46 escolas, aproximadamente 2700 crianças, estratificadas por área e por tipo de oferta escolar. O estudo longitudinal contou com 3 ondas de coleta de dados sobre as crianças: duas coletas em 2017, no início e final do ano letivo, aos 4/5 anos de idade, e uma coleta no final do ano letivo de 2018, aos 5/6 anos. Para mais detalhes sobre a amostra ver Bartholo e outros $(2019 ; 2020)$. A figura abaixo ilustra o desenho do estudo longitudinal.

Nas ondas 2 e 3 foram incluídas na amostra crianças que entraram durante a pré-escola nas escolas da amostra. $\mathrm{O}$ desenvolvimento cognitivo dos alunos foi estimado a cada onda da pesquisa, utilizando uma adaptação do instrumento Performance Indicators in Primary

${ }^{2}$ Utilizamos para tanto as categorias criadas pelo Instituto Brasileiro de Geografia e Estatística (IBGE).

${ }^{3} \mathrm{O}$ estudo de Carvalho (2005) explora questões relacionadas à identidade da raça para alunos no início do ensino fundamental. 
Schools (PIPS), uma ferramenta desenvolvida por pesquisadores da Durham University, na Inglaterra (Tymms, 1999).

\begin{tabular}{ccc} 
Pré-escola I & Pré-escola I & Pré-escola II \\
$\mathrm{O}_{1}$ & $\mathrm{O}_{2}$ & $\mathrm{O}_{3}$ \\
4 anos & $4 / 5$ anos & $5 / 6$ anos \\
\hline Março/2017 & & Dezembro/2018
\end{tabular}

Figura 1. Desenho do estudo longitudinal

Fonte: Elaboração própria dos autores.

O instrumento mede duas dimensões, Linguagem e Matemática, sendo estas compostas pelas seguintes subdimensões: “a) Escrita; b) Vocabulário; c) Ideias sobre leitura - avalia conceitos sobre diferentes impressos; c) Consciência fonológica; d) Identificação de letras; e) Reconhecimento de palavras e leitura; f) Ideias sobre matemática; g) Contagem e números; h) Adição e subtração sem símbolos; i) Identificação de formas; j) Identificação de números" (Koslinski e Bartholo, 2019, p. 293).

Os escores de Linguagem e Matemática foram estimados a partir dos itens do teste cognitivo da adaptação do teste PIPS empregando medidas de Rasch (Boone, 2006), com base no software Winstep.

Quadro 1. Descrição das variáveis analisadas e suas fontes

\begin{tabular}{|c|c|c|c|}
\hline NoME & TIPO & DESCRIÇÃO & FONTE \\
\hline \multicolumn{4}{|c|}{ Variáveis Dependentes } \\
\hline Linguagem & Contínua & Medida de linguagem na onda 2 ou 3 & Teste cognitivo \\
\hline Matemática & Contínua & Medida de matemática na onda 2 ou 3 & Teste cognitivo \\
\hline \multicolumn{4}{|c|}{ Variáveis Explicativas } \\
\hline \multicolumn{4}{|c|}{ Nível 2 - escola } \\
\hline $\begin{array}{l}\text { NSE } \\
\text { (escola) }\end{array}$ & Contínua & $\begin{array}{l}\text { Média do indicador de NSE agregado por } \\
\text { escola }\end{array}$ & $\begin{array}{l}\text { Questionário } \\
\text { responsáveis e } \\
\text { SGA/SME }\end{array}$ \\
\hline \multicolumn{4}{|c|}{ Nível 1 - criança } \\
\hline Linguagem & Contínua & Medida de linguagem na onda 1 ou 2 & Teste cognitivo \\
\hline Matemática & Contínua & Medida de matemática na onda 1 ou 2 & Teste cognitivo \\
\hline $\begin{array}{l}\text { NSE } \\
\text { (criança) }\end{array}$ & Contínua & $\begin{array}{l}\text { Indicador de nível socioeconômico da } \\
\text { criança, calculado utilizando modelagem de } \\
\text { Rasch com itens sobre escolaridade dos } \\
\text { pais, posse de bens, densidade domiciliar e } \\
\text { pobreza (acesso a programas de } \\
\text { transferência de renda) }\end{array}$ & $\begin{array}{l}\text { Quest. } \\
\text { Responsáveis e } \\
\text { SGA/SME }\end{array}$ \\
\hline Sexo & Dicotômica & $\begin{array}{l}\text { Indica o sexo da criança }(0=\text { Menina; } 1= \\
\text { Menino) }\end{array}$ & SGA/SME \\
\hline Idade & Contínua & $\begin{array}{l}\text { Indica a idade em anos da criança na onda } 1 \text {, } \\
2 \text { ou } 3\end{array}$ & SGA/SME \\
\hline SEN & Dicotômica & $\begin{array}{l}\text { Indica se criança é portadora de } \\
\text { necessidades educacionais especiais }\end{array}$ & \\
\hline
\end{tabular}

Fonte: Elaboração dos autores. 
O quadro 1 apresenta as variáveis utilizadas nos modelos multinível para estimar o efeito par (compositional effect) para o primeiro e segundo ano na escola. A interpretação dos modelos foca no efeito do indicador de nível socioeconômico agregado para a escola (nível dois do modelo multinível). Para minimizar a perda de casos ao longo dos dois anos do estudo, os modelos estimaram separadamente o efeito par (compositional effect) no primeiro e em seguida no segundo ano na escola.

Além dos dados cognitivos das crianças, o estudo longitudinal coletou informações do contexto familiar durante as ondas 2 e 3 a partir de questionários para pais e responsáveis. Os dados demográficos das crianças (idade, cor, sexo) e a complementação de dados sobre o contex to socioeconômico da família (escolaridade dos pais e participação do programa bolsa-família) foram obtidos a partir do Sistema de Gestão Acadêmica da Secretaria Municipal de Educação (SGA/SME).

Para calcular o efeito par (compositional effect) estimamos modelos utilizando a variável de NSE do nível individual agregadas para o nível da escola. Os modelos de regressão multinível, em que o nível 1 se refere ao indivíduo/criança e o nível 2 à escola, incluíram as variáveis no nível 1 centrados na grande média. Os modelos mais simples, que incluíram somente a variável de NSE no nível 1 e o NSE agregado por escola no nível 2, podem ser expressos pela equação abaixo:

Modelo nível 1:

$$
\text { Medida Cognitiva } a_{i j}=\beta_{0 j}+\beta_{1 j} *\left(\mathrm{NSE}_{\mathrm{ij}}\right)+\mathrm{r}_{\mathrm{ij}}
$$

Modelo nível 2:

$$
\begin{aligned}
& B_{o j}=\gamma_{o o}+\gamma_{o t} *(\text { NSE escola } j)+u_{o j}, \\
& \boldsymbol{B}_{l j}=\boldsymbol{\gamma}_{i o}
\end{aligned}
$$

Os resultados do efeito par/compositional effect são observados diretamente a partir do coeficiente de NSE agregado pela escola $\left(\gamma_{\mathrm{O} 1}\right)$, o efeito intra-grupo a partir do coeficiente do NSE no nível $1\left(\gamma_{10}\right)$ e o efeito entre-grupos é obtido a partir da soma dos dois efeitos, intra-grupo e efeito par/compositional effect (Harker e Tymms, 2010). Modelos subsequentes incluíram, além das variáveis de NSE no nível 1 e agregada no nível 2, outros controles relacionados às características demográficas da criança (sexo, idade e necessidades educacionais especiais) e controle da medida cognitiva anterior. Os quadros no corpo do texto apresentam os coeficientes, erro padrão e effect sizes, calculados utilizando a metodologia proposta por Tymms (2004), somente para o compositional effect, efeito intra-grupo, além da soma dos dois coeficientes que expressam o efeito entregrupos, dos modelos estimados". O artigo apresenta os coeficientes em effect size para relatar a diferença entre dois grupos, para maiores detalhes ver Tymms (2004). Um número crescente de estudos no campo da educação opta por reportar seus resultados em effect size e há extenso debate sobre as melhores formas de interpretação (Higgins, Kokotsaky e Coe, 2012).

Também estimamos modelos similares com a inserção da variável cor (preto) no nível 1 e agregada no nível 2. Trabalho anterior realizados com dados do ensino fundamental

\footnotetext{
${ }^{4}$ Os quadros completos no anexo do artigo.
} 
observaram efeito da composição racial da escola (Bartholo e Costa, 2016). No entanto, os modelos estimados para a pré-escola apresentaram resultados inconsistente e os coeficientes estimados para cor não apresentaram significância estatística em nenhum dos modelos estimados. Frente as limitações da variável cor anteriormente relatadas, optamos por não apresentar os resultados desses modelos.

\section{Padrões de segregação no início da escolarização formal}

Diversos estudos no Brasil calcularam indicadores de segregação escolar utilizando dados secundários do Censo Escolar Inep (Instituto Nacional de Estatística Educacional) ou bases administrativas de secretarias municipais ou estaduais de ensino (Bartholo, 2013; Bartholo e Costa, 2018; Mendes, 2017). No entanto, os estudos citados não calcularam a segregação para o período compreendido como pré-escola, crianças entre 4 e 5 anos, período obrigatório de escolarização no Brasil desde 2009. Alteração foi feita na Lei de Diretrizes e Bases (LDB) por meio da lei número 12.796.

O quadro 2 apresenta os índices de segregação entre 2006 e 2013 utilizando o GS para duas características de desvantagem, a saber, educação dos pais e cor/raça e compara os padrões de segregação entre a pré-escola e o $1^{\circ}$ ano do ensino fundamental.

Quadro 2. Padrões de segregação escolar na pré-escola e no $1^{\circ}$ ano do ensino fundamental (2006-2013)

\begin{tabular}{lcccccccc}
\hline & $\mathbf{2 0 0 6}$ & $\mathbf{2 0 0 7}$ & $\mathbf{2 0 0 8}$ & $\mathbf{2 0 0 9}$ & $\mathbf{2 0 1 0}$ & $\mathbf{2 0 1 1}$ & $\mathbf{2 0 1 2}$ & $\mathbf{2 0 1 3}$ \\
\hline Pré-escola Cor & 17,5 & 18 & 18,5 & 18 & 18,5 & 19 & 18,5 & 18,5 \\
$1^{\circ}$ Ano Cor & 19,5 & 19 & 20 & 20 & 19 & 19 & 20,5 & 20,5 \\
Pre-Escola EducEF & 24 & 24 & 22 & 22,5 & 24,5 & 23,5 & 24 & 25 \\
10 Ano EducEF & 24 & 23 & 22,5 & 22 & 21 & 22 & 22,5 & 23 \\
Pre-Escola EducEM & 11 & 11,5 & 10 & 10,5 & 12 & 12,5 & 12 & 13 \\
10 Ano EducEM & 10,5 & 10,5 & 10,5 & 10 & 10 & 11,5 & 12 & 12 \\
\hline
\end{tabular}

Fonte: Elaboração própria dos autores.

Os dados descritos sugerem relativa estabilidade dos padrões de segregação na pré-escola ao longo do período estudado. Ainda mais importante, é possível observar grande similaridade dos níveis de segregação descritos para a pré-escola e $1^{\circ}$ ano do ensino fundamental. Esse resultado reforça a hipótese de que os fatores que influenciam a segregação escolar nas duas etapas seriam semelhantes. Podemos destacar as regras de matrícula, que são idênticas para ambas as faixas etárias. No entanto, duas diferenças importantes poderiam afetar os padrões de segregação escolar observados. A primeira é a proporção de crianças matriculadas na rede pública na pré-escola e no ensino fundamental na cidade do Rio de Janeiro. A segunda é o total de escolas na rede pública de ensino que ofertam turmas de pré-escola e do $1^{\circ}$ ano do ensino fundamental. Ambas as características poderiam, potencialmente, alterar os níveis de segregação escolar mensurados.

O número total de escolas e sua relação com os padrões de segregação escolar já foi explorado por Bartholo (2014) em estudo na rede pública da cidade do Rio de Janeiro. Esse estudo indicou uma redução importante nos níveis de segregação escolar mensurados na transição entre o primeiro e segundo segmentos do ensino fundamental, período em que grande parte dos alunos mudam de escola. Nessa transição, pode ser observada uma 
redução média de $45 \%$ no total de escolas, o que aumenta a chance de as escolas apresentarem perfil discente mais diversificado. Trata-se, portanto, de um efeito probabilístico, observado em outros sistemas educacionais quando ocorre uma diminuição importante no número de escolas (Bartholo, 2014). O quadro 3 apresenta o total de escolas que oferecem matrículas na pré-escola e $1^{\circ}$ ano do ensino fundamental entre 2007 e 2013.

Quadro 3. Número de escola e de alunos/crianças matriculados na rede municipal do Rio de Janeiro por etapa

\begin{tabular}{lccccccc}
\hline & $\mathbf{2 0 0 7}$ & $\mathbf{2 0 0 8}$ & $\mathbf{2 0 0 9}$ & $\mathbf{2 0 1 0}$ & $\mathbf{2 0 1 1}$ & $\mathbf{2 0 1 2}$ & $\mathbf{2 0 1 3}$ \\
\hline $\begin{array}{l}\text { N escolas c/ oferta de pré- } \\
\text { escola }\end{array}$ & 731 & 723 & 721 & 708 & 697 & 728 & 709 \\
\hline $\begin{array}{l}\text { N escolas c/ oferta dos } \\
\text { anos iniciais do EF }\end{array}$ & 870 & 874 & 896 & 852 & 877 & 840 & 833 \\
\hline
\end{tabular}

Fonte: Sinopse estatística da educação básica (MEC/INEP, 2007-2013).

Os dados evidenciam um aumento proporcional de aproximadamente $18 \%$ no número de escolas na transição entre a pré-escola e o ensino fundamental. Esse cenário poderia, pelo menos em tese, aumentar os níveis de segregação escolar no sistema educacional, no entanto, os dados não sugerem esse efeito.

Na cidade do Rio de Janeiro, os dados do Censo Populacional de 2010 indicavam boa adesão das famílias e um aumento da oferta de vagas na pré-escola e no ensino fundamental: a taxa de matrícula para crianças e adolescentes de 6 a 14 (Ensino Fundamental) era de $97 \%$, enquanto a taxa de matrícula de crianças de 4 a 5 anos na educação infantil era de $88 \%$. Além disso, no período estudado, a rede privada era responsável por uma proporção maior de matrículas na etapa da pré-escola (variando de 27 a $45 \%$ ), se comparada com a proporção do ensino fundamental (variando de 18 a 32\%). Desta forma, esperávamos encontrar uma maior homogeneidade do alunado e menor segregação escolar na etapa da pré-escola do que entre as escolas que atendem alunos matriculados no $1^{\circ}$ ano do ensino fundamental. $\mathrm{O}$ gráfico 1 apresenta a proporção de matriculas na rede pública, considerando todas as crianças matriculadas nas duas etapas de ensino, pré-escola e $1^{\circ}$ ano do ensino fundamental.

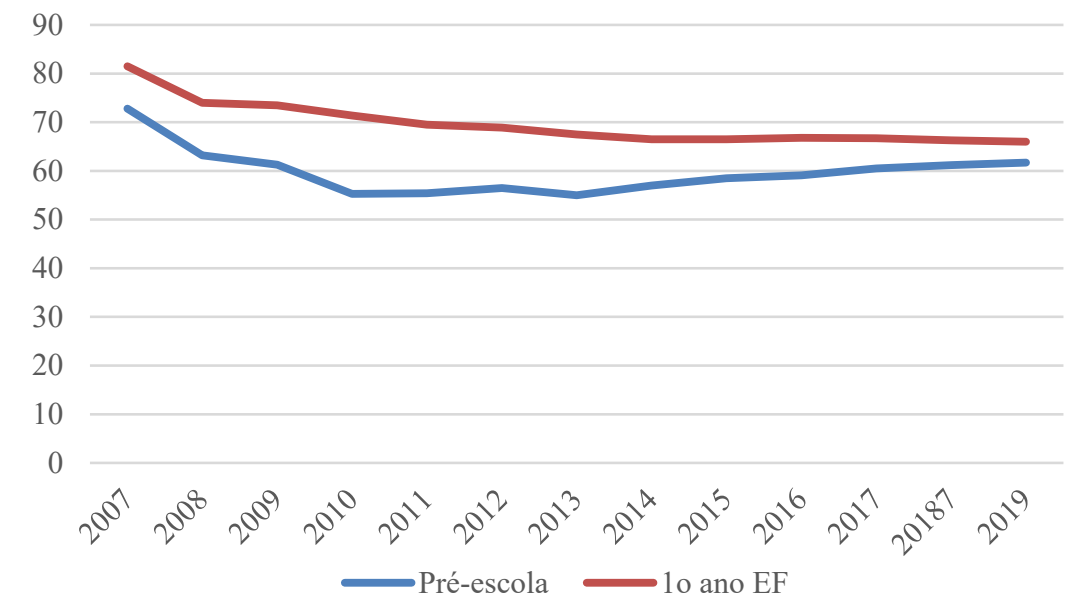

Gráfico 1. Proporção de matrículas na rede pública para a pré-escola e $1^{\circ}$ ano do ensino fundamental no município do Rio de Janeiro

Fonte: Censo Escolar, Inep. Elaboração própria dos autores. 
É interessante notar que no período entre 2007 e 2019 a proporção de alunos matriculados na rede pública diminui, o que evidencia um aumento proporcional de matriculas na rede privada tanto na pré-escola quanto no $1^{\circ}$ ano do ensino fundamental. Esse fenômeno merece ser estudado com mais detalhes por pesquisadores do campo educacional e pode estar associado a percepção sobre a qualidade do ensino público e, também, queda diferencial na taxa de natalidade de diferentes grupos de perfil socioeconômico no Brasil.

A proporção de crianças matriculadas no ensino fundamental e na pré-escola na rede pública sugere que uma parte maior da demanda durante a pré-escola era atendida pela rede privada. Isso pode ser explicado pela não obrigatoriedade da oferta da pré-escola até 2009 e a subsequente estabelecimento de metas para universalização do acesso ao sistema escolar para crianças entre 4 e 5 anos até o ano de 2016.

Esse fator é importante porque sugere um duplo efeito na transição da pré-escola para o ensino fundamental. O primeiro é uma migração de alunos que estavam na rede privada para a rede pública, possivelmente diante da falta de vagas na rede pública na etapa da préescola. Um segundo efeito é o ingresso de crianças que estavam fora da escola, portanto não frequentaram a pré-escola, e passaram a frequentar o $1^{\circ}$ ano do ensino fundamental. Essas duas mudanças têm o potencial de alterar de forma significativa a proporção de alunos em desvantagem potencial na rede pública quando comparamos a pré-escola com o $1^{\circ}$ ano do ensino fundamental. Não é claro se os alunos que estavam fora da pré-escola eram de perfil desfavorecido, mas os dados da Amostra do Censo Populacional de 2010 para o Estado do Rio de Janeiro sugerem que sim. Por exemplo, a taxa de matrícula de crianças de 4 e 5 anos era equivalente a $88 \%$ para as crianças brancas e a $83 \%$ para crianças pretas e pardas, reforçando a ideia de que crianças mais vulneráveis teriam menos acesso à essa etapa de escolarização.

\section{Efeito da composição do alunado nos primeiros dois anos na escola}

Nessa etapa serão apresentados os resultados dos modelos hierárquicos que estimam o aprendizado das crianças ao longo do primeiro e do segundo ano na escola. Dividimos as análises em dois conjuntos. O primeiro foca no indicador de nível socioeconômico (NSE) construído utilizando dados da entrevista com os responsáveis e dados secundários do SGA/SME no primeiro ano da escola e o segundo replica as mesmas análises para o segundo ano na escola. A replicação ajuda a observar um padrão nos resultados e refutar, mesmo que parcialmente, o que Harker e Tymms (2004) denominaram efeito fantasma/ phantom effect.

Os coeficientes das regressões multínivel são apresentados no quadro 4. Os modelos apresentam resultados para matemática e linguagem, separados para o primeiro e segundo ano na escola, inserindo apenas a variável de NSE no nível do indivíduo e o NSE agregado para a escola no nível dois do modelo. Os resultados sugerem um efeito maior da composição do alunado para linguagem, em especial no segundo ano da pré-escola. Esse resultado corrobora o achado de Harker e Tymms (2004) e Bartholo e Costa (2016). É importante destacar que a partição da variância observada no modelo nulo sugere que há pouca variação entre escolas. $\mathrm{O}$ valor observado no segundo ano na escola é ligeiramente maior do que o observado no primeiro ano, no entanto, ainda pequeno. 
Quadro 4. Efeito par (compositional effect) do NSE agregado em Matemática e Linguagem

\begin{tabular}{|c|c|c|c|c|c|c|c|c|}
\hline & \multicolumn{2}{|c|}{$\begin{array}{c}\text { MATEMÁTICA } \\
1^{\circ} \text { ANO }\end{array}$} & \multicolumn{2}{|c|}{$\begin{array}{c}\text { MATEMÁTICA } \\
\mathfrak{2}^{\circ} \text { ANO }\end{array}$} & \multicolumn{2}{|c|}{$\begin{array}{c}\text { LINGUAGEM } \\
1^{\circ} \text { ANO }\end{array}$} & \multicolumn{2}{|c|}{$\begin{array}{c}\text { LINGUAGEM } \\
\mathbf{2}^{\circ} \text { ANO }\end{array}$} \\
\hline & $\begin{array}{l}\text { Coef. } \\
\text { (ES) }\end{array}$ & $\begin{array}{l}\text { Err. } \\
\text { pad. }\end{array}$ & $\begin{array}{l}\text { Coef. } \\
\text { (ES) }\end{array}$ & $\begin{array}{l}\text { Err. } \\
\text { pad. }\end{array}$ & $\begin{array}{l}\text { Coef. } \\
\text { (ES) }\end{array}$ & $\begin{array}{l}\text { Err. } \\
\text { pad. }\end{array}$ & $\begin{array}{l}\text { Coef. } \\
(\mathrm{ES})\end{array}$ & $\begin{array}{l}\text { Err. } \\
\text { pad. }\end{array}$ \\
\hline Intra & $\begin{array}{l}0,311^{\text {***** }} \\
(0,481)\end{array}$ & 0,028 & $\begin{array}{c}0,301^{* * * *} \\
(0,404)\end{array}$ & 0,031 & $\begin{array}{l}0,084^{* *} \\
(0,419)\end{array}$ & 0,029 & $\begin{array}{c}0,172^{* * * *} \\
(0,379)\end{array}$ & 0,020 \\
\hline Entre & 0,428 & & 0,425 & & 0,253 & & 0,304 & \\
\hline Comp. & $\begin{array}{l}0,127^{* * *} \\
(0,196)\end{array}$ & 0,039 & $\begin{array}{l}0,124^{* * *} \\
(0,166)\end{array}$ & 0,040 & $\begin{array}{c}0,169^{* * * *} \\
(0,207)\end{array}$ & 0,018 & $\begin{array}{c}0,133^{\text {***** }} \\
(0,293)\end{array}$ & 0,031 \\
\hline
\end{tabular}

Nota: ${ }^{* * *} \mathrm{p}<0,001,{ }^{* *} \mathrm{p}<0,01$.

Fonte: Elaboração própria dos autores.

O modelo apresentado no quadro 5 é muito simples e há possibilidade real que a medida de NSE no nível individual e agregado para a escola esteja explicando parte da variação de outras dimensões ausentes no modelo. Por exemplo, idade, sexo ou mesmo uma medida inicial de desenvolvimento. Os modelos subsequentes inserem novas covariáveis para observar se os efeitos observados no NSE no nível um e dois do modelo continuam explicando parte da variação no aprendizado dos alunos. $\mathrm{O}$ quadro 5 apresenta os coeficientes de um modelo que introduz três novas variáveis, a saber, sexo, idade e necessidades educacionais especiais.

Quadro 5. Efeito par (compositional effect) do NSE agregado, controlando por outras variáveis do nível 1

\begin{tabular}{|c|c|c|c|c|c|c|c|c|}
\hline & \multicolumn{2}{|c|}{$\begin{array}{c}\text { MATEMÁTICA } \\
1^{\circ} \text { ANO } \\
\end{array}$} & \multicolumn{2}{|c|}{$\begin{array}{c}\text { MATEMÁtiCA } \\
\mathbf{2}^{\circ} \text { ANO } \\
\end{array}$} & \multicolumn{2}{|c|}{$\begin{array}{c}\text { LINGUAGEM } \\
\mathbf{1}^{\circ} \text { ANO }\end{array}$} & \multicolumn{2}{|c|}{$\begin{array}{c}\text { LINGUAGEM } \\
\mathbf{2}^{\circ} \text { ANO } \\
\end{array}$} \\
\hline & $\begin{array}{c}\text { Coef. } \\
\text { (ES) }\end{array}$ & $\begin{array}{l}\text { Err. } \\
\text { pad. }\end{array}$ & $\begin{array}{l}\text { Coef. } \\
\text { (ES) }\end{array}$ & $\begin{array}{l}\text { Err. } \\
\text { pad. }\end{array}$ & $\begin{array}{l}\text { Coef. } \\
\text { (ES) }\end{array}$ & $\begin{array}{l}\text { Err. } \\
\text { pad. }\end{array}$ & $\begin{array}{l}\text { Coef. } \\
\text { (ES) }\end{array}$ & $\begin{array}{l}\text { Err. } \\
\text { pad. }\end{array}$ \\
\hline Intra & $\begin{array}{c}0,319^{* * * *} \\
(0,523)\end{array}$ & 0,026 & $\begin{array}{c}0,321^{* * * *} \\
(0,461)\end{array}$ & 0,029 & $\begin{array}{c}0,176^{* * * *} \\
(0,468)\end{array}$ & 0,016 & $\begin{array}{l}0,191^{* * * *} \\
(0,453)\end{array}$ & 0,018 \\
\hline Entre & 0,466 & & 0,474 & & 0,273 & & 0,339 & \\
\hline Comp. & $\begin{array}{c}0,147^{* * * *} \\
(0,241)\end{array}$ & 0,032 & $\begin{array}{c}0,153^{* * * *} \\
(0,220)\end{array}$ & 0,036 & $\begin{array}{c}0,097^{* * * *} \\
(0,257)\end{array}$ & 0,026 & $\begin{array}{c}0,148^{* * * *} \\
(0,352)\end{array}$ & 0,032 \\
\hline
\end{tabular}

Nota: ${ }^{* * *} \mathrm{p}<0,001$.

Fonte: Elaboração própria dos autores.

Os resultados dos modelos sugerem que há efeito da composição do alunado para matemática e linguagem ao longo dos dois primeiros anos na escola. É interessante notar que, mesmo após a introdução de outras variáveis de controle, o tamanho do efeito observado no NSE agregado tornou-se ligeiramente maior em todos os modelos estimados. No entanto, esses modelos ainda apresentam uma grande fragilidade por não apresentarem uma medida inicial de controle sobre o desenvolvimento do aluno. $\mathrm{O}$ quadro 6 apresenta o modelo final, incluindo todas as covariáveis descritas anteriormente e uma medida inicial sobre o desenvolvimento dos alunos em matemática ou linguagem. 
Quadro 6. Efeito par (compositional effect) do NSE agregado, controlando por variáveis do nível 1 e desempenho anterior

\begin{tabular}{|c|c|c|c|c|c|c|c|c|}
\hline & \multicolumn{2}{|c|}{$\begin{array}{c}\text { MATEMÁTICA } \\
1^{\circ} \text { ANO }\end{array}$} & \multicolumn{2}{|c|}{$\begin{array}{c}\text { MATEMÁTICA } \\
\mathfrak{2}^{\circ} \text { ANO }\end{array}$} & \multicolumn{2}{|c|}{$\begin{array}{c}\text { LINGUAGEM } \\
1^{\circ} \text { ANO }\end{array}$} & \multicolumn{2}{|c|}{$\begin{array}{c}\text { LINGUAGEM } \\
\boldsymbol{2}^{\circ} \text { ANO }\end{array}$} \\
\hline & $\begin{array}{l}\text { Coef. } \\
\text { (ES) }\end{array}$ & $\begin{array}{l}\text { Err. } \\
\text { pad. }\end{array}$ & $\begin{array}{l}\text { Coef. } \\
\text { (ES) }\end{array}$ & $\begin{array}{l}\text { Err. } \\
\text { pad. }\end{array}$ & $\begin{array}{l}\text { Coef. } \\
\text { (ES) }\end{array}$ & $\begin{array}{l}\text { Err. } \\
\text { pad. }\end{array}$ & $\begin{array}{l}\text { Coef. } \\
\text { (ES) }\end{array}$ & $\begin{array}{l}\text { Err. } \\
\text { pad. }\end{array}$ \\
\hline Intra & $\begin{array}{l}0,120^{* * * *} \\
(0,270)\end{array}$ & 0,020 & $\begin{array}{l}0,042^{+} \\
(0,093)\end{array}$ & 0,024 & $\begin{array}{l}0,061^{* * * *} \\
(0,210)\end{array}$ & 0,014 & $\begin{array}{l}0,052^{* * * *} \\
(0,178)\end{array}$ & 0,014 \\
\hline Entre & 0,174 & & 0,098 & & & & 0,122 & \\
\hline Compo. & $\begin{array}{l}0,055^{+} \\
(0,123)\end{array}$ & 0,032 & $\begin{array}{l}0,055^{*} \\
(0,121)\end{array}$ & 0,025 & $\begin{array}{l}0,066^{*} \\
(0,225)\end{array}$ & 0,025 & $\begin{array}{l}0,070^{*} \\
(0,241)\end{array}$ & 0,026 \\
\hline
\end{tabular}

Nota: ${ }^{* * *} \mathrm{p}<0,001,{ }^{* *} \mathrm{p}<0,01,{ }^{*} \mathrm{p}<0,05,{ }^{+} \mathrm{p}<0,10$.

Fonte: Elaboração própria dos autores.

Para o primeiro ano na escola, os coeficientes sugerem um efeito par (compositional effect) para a medida de linguagem com o indicador de NSE agregado para a escola significativo a 0,05. Para matemática, a introdução das demais covariáveis, em especial a medida inicial, não alteram a direção do resultado, porém o indicador diminui em tamanho do efeito a 0,10. Esse é um bom exemplo do que Harker e Tymms (2004) chamam de phantom effect, quando o efeito observado desaparece, em especial após introdução de medida de controle sobre o ponto e partida dos alunos no desenvolvimento cognitivo. O resultado reforça a importância de modelos de valor agregado (value-added models) para pesquisas sobre efeito da escola, impacto de programas educacionais ou mesmo efeito da composição do alunado. A ausência de medidas longitudinais sobre o desenvolvimento das crianças pode levar o pesquisador a resultados e interpretações equivocadas.

Para o segundo ano na escola, os resultados sugerem que há efeito da composição do alunado sobre o aprendizado em linguagem e matemática. $\mathrm{O}$ tamanho do efeito para ambos os modelos diminui, porém permanecem estatisticamente significativo a 0,05 , mesmo no modelo final com controle do ponto de partida no teste cognitivo. Cabe reforçar que no segundo ano da pré-escola observamos um aumento da variação entre escolas, o que em tese pode explicar o fato de encontramos um efeito da composição do alunado para linguagem e matemática. Os resultados preliminares reforçam o achado de Bartholo e Costa (2016) e sugerem a existência de um efeito da composição do alunado para linguagem. Para matemática, os resultados são menos claros e sugerem que há apenas efeito da composição do alunado no segundo ano na escola.

Os resultados apresentados no artigo, utilizaram dados apenas para os alunos matriculados na rede pública. Essa é uma limitação importante dos resultados. É razoável presumir que, ao incorporar os dados dos alunos matriculados na rede privada, os resultados seriam diferentes. Bartholo e Costa (2018) evidenciaram que os níveis de segregação quando calculados apenas com alunos matriculados na rede pública subestimam o fenômeno. A explicação é que há claro viés no perfil socioeconômico dos alunos quando comparamos as matrículas nas redes pública e privada. O mesmo argumento pode ser construído para pensarmos o efeito da composição do alunado. É provável que o tamanho do efeito seja maior do que o descrito no artigo. Ao estimar o efeito par apenas com uma parte mais homogênea do alunado matriculada na rede pública, uma parte importante da variação entre escolas não é captada no modelo. 
Os resultados reforçam a necessidade de qualificar o debate sobre as desigualdades educacionais no início da escolarização obrigatória. Regras de matrícula claras, processos transparentes e políticas focadas em tornar as escolas públicas mais atraentes podem ajudar reverter ao menos parte dos padrões de segregação escolar observados (Rosistolato, et al., 2019). Isto porque, para além das regras de matrícula, como o presente estudo trata de etapas do sistema escolar que atendem a crianças pequenas e com menor mobilidade no que diz respeito à escolha e acesso ao estabelecimento escolar, o fenômeno da segregação escolar sofre maior influência dos padrões de segregação residencial característicos das grandes cidades brasileiras (Érnica e Batista, 2012; Koslinski, Alves e Lange, 2013).

\section{Conclusão}

Há um crescente debate sobre a importância da educação infantil, e de forma mais específica da pré-escola, como política pública capaz de diminuir desigualdades educacionais e sociais. Analisar a distribuição de oportunidades educacionais nesse período do processo de escolarização é importante para pensarmos a equidade do sistema.

Os dados analisados no artigo apresentam a primeira tentativa de medir o fenômeno da segregação escolar ao longo do tempo para a pré-escola (período obrigatório do processo de escolarização) e estimar o efeito par para os primeiros dois anos na escola no contexto brasileiro. Os resultados preliminares sugerem que os padrões de segregação escolar observados na pré-escola são semelhantes ao descritos no $1^{\circ}$ ano do ensino fundamental. Não há, portanto, grande impacto nos padrões de segregação na transição entre as etapas de ensino. Estudos futuros devem incorporar dados da rede privada para a etapa da préescola e descrever o impacto da estratificação entre as redes pública e privada. Não obstante, os resultados descritos reforçam a importância do estudo da temática da segregação escolar e revelam pela primeira vez o tamanho do fenômeno para a faixa etária entre 4 e 5 anos em uma grande cidade do Brasil.

Os modelos para estimar o efeito da composição social das escolas (compositional effect) no aprendizado dos alunos sugerem que há efeito, com resultados mais consistentes para a medida de linguagem. Ressaltamos que as medidas de nível socioeconômico utilizadas no presente trabalho -elaboradas a partir de dados coletados diretamente com pais/responsáveis das crianças- são mais detalhadas, com menos dados faltantes e, portanto, mais confiáveis que as medidas presentes em estudos que utilizam dados secundários, elaboradas a partir da resposta dos alunos. A proposta de apresentar modelos com um crescente número de variáveis de controle reforça a importância de pesquisas longitudinais com duas ou mais medidas sobre o desenvolvimento das crianças. É importante destacar ainda que é provável que a ausência dos alunos matriculados na rede privada, subestime o fenômeno do efeito da composição do alunado. Estudos futuros devem recalcular o modelo incorporando essa parcela da população, bem como observar possíveis alterações no padrão da segregação escolar frente a persistência da tendência, observada desde 2013, de aumento das matrículas da etapa da pré-escola na rede pública

Estudos futuros devem explorar ainda outras características de desvantagem potencial na tentativa de estimar o efeito par, como por exemplo, cor/raça ou bairro de moradia considerando crianças que vivem em favelas. Além disso, podem utilizar controles de outras variáveis relacionadas tanto ao professor, como autoeficácia, quanto aos processos 
escolares, como clima escolar, que permitam diferenciar o efeito da composição do alunado dos efeitos relacionados ao contexto da escola/sala de aula.

\section{Referências}

Alves, L., Batista, A. A. G., Ribeiro, V. M. e Érnica, M. (2015). Seleção velada em escolas públicas: Práticas, processos e princípios geradores. Educação e Pesquisa, 41, 37-152. https://doi.org/10.1590/S1517-97022015011488.

Bartholo, T. L. (2013). Measuring between-school segregation in an open enrollment system: The case of Rio de Janeiro. Journal of School Choice, 7, 353-371. https://doi.org/10.1080/15582159.2013.808937

Bartholo, T. L. (2014). Segregação escolar na cidade do Rio de Janeiro: Análise da movimentação de estudantes. Estudos em Avaliação Educacional, 25, 242. https://doi.org/10.18222/eae255820142927

Bartholo, T. L. e Costa, M. (2014). Turnos e segregação escolar: Discutindo as desigualdades intraescolares. Cadernos de Pesquisa, 44, 670-692. https://doi.org/10.1590/198053142771

Bartholo, T. L. e Costa, M. (2016). Evidence of a school composition effect in Rio de Janeiro public schools. Ensaio, 24, 498-521. https://doi.org/10.1590/S0104-40362016000300001

Bartholo, T. L. e Costa, M. (2018). Patterns of school segregation in Brazil: Inequalities and education policy. En X. Bonal e C. Bellei (Orgs.), Understanding school segregation: Patterns, causes and consequences of spatial inequalities in education (pp. 65-82). Bloomsbury Academic. https://doi.org/10.5040/9781350033542.ch-004

Bartholo, T. L., Koslinski, M. C., Costa, M. e Barcellos, T. M. (2019). What do children know upon entry to pre-school in Rio de Janeiro? Ensaio, 7, 1-22. https://doi.org/10.1590/s0104-40362019002702071

Bartholo, T. L., Koslinski, M. C., Costa, M., Tymms, P. B., Merrell, C. e Barcellos, T. M. (2020). The use of cognitive instruments for research in early childhood education: constraints and possibilities in the Brazilian context. Pro-Posições, 31, e20180036. https://doi.org/10.1590/1980-6248-2018-0036

Boone, W. J. (2006). Rasch analysis for instrument development: Why, when, and how? Erin Dolan.

Bruel, A. L. e Bartholo, T. L. (2012). Desigualdade de oportunidades educacionais na rede pública municipal do Rio de Janeiro: Transição entre os segmentos do ensino fundamental. Revista Brasileira de Educação, 17, 303-328. https://doi.org/10.1590/s1413-24782012000200004

Carvalho, M. P. (2005). Quem é negro, quem é branco: Desempenho escolar e classificação racial de alunos. Revista Brasileira de Educação, 28, 77-95. https://doi.org/10.1590/S1413-24782005000100007.

Carvalho Filho, J. L. (2016). Segregação espacial e segregação escolar: Notas para uma sociologia da distribuição espacial e social dos estabelecimentos de ensino. Mediações, 21(2), 359-380. https://doi.org/10.5433/2176-6665.2016v2 1n2p359

Castro. C. M. (2016). Avaliação do aprendizado no ciclo de alfabetização de alunos da rede municipal do Rio de Janeiro (Dissertação de Mestrado). Escola Nacional de Ciências Estatísticas IBGE.

Denton, N. A. (1996). The persistence of segregation: Links between residential segregation and school segregation. Minnesota Law Review, 80, 795-824.

Duncan, O. D. e Duncan, B. (1955). A methodological analyses of segregation indexes. American Sociological Review, 20(2), 210-217. 
Érnica, M. e Batista, A. A. G. (2012). A escola, a metrópole, e a vizinhança vulnerável. Cadernos de Pesquisa, 42, 640-666. https://doi.org/10.1590/So100-15742012000200016

Frankenberg, E. (2013) The role of residential segregation in contemporary school segregation. Education and Urban Society, 45(5), 548-570. https://doi.org/10.1177/0013124513486288

Goldhaber, D. (1999). School choice: An examination of the empirical evidence on achievement, parental decision making and equity. Educational Researcher, 28, 16-25. https://doi.org/10.3102/0013189X028009016

Gorard, S. e See, B. H. (2013) Overcoming disadvantage in education. Routledge.

Gorard, S., Taylor, C. e Fitz, J. (2003). Schools, markets and choice policies. Routledge. https://doi.org/10.4324/9780203609040

Harker, R. e Tymms, B. (2004). The effects of student composition on school outcomes. School effectiveness and school improvement. An International Journal of Research, Policy and Practice, 15(2), 177-199. https://doi.org/ 10.1076/sesi.15.2.177.30432

Harris, R. (2012). Local indices of segregation with application to social segregation between London's secondary schools. Environment and Planning, 44, 669-687. https://doi.org/10.1068/a44317

Higgins, S., Kokotsaky, D. e Coe, R. (2012). The teaching and learning toolkit: Technical appendices. Education Endowment Foundation.

Hutchens, R. (2004). One measure of segregation. International Economic Review, 45(2), 555-578. https://doi.org/10.1111/j.1468-2354.2004.00136.x

Koslinski, M. C. e Carvalho, J. T. (2015). Escolha, seleção e segregação nas escolas municipais do Rio de Janeiro. Cadernos de Pesquisa, 45(158), 916-942. ttps://doi.org/10.1590/198053143352

Koslinski, M. C., Alves, F. e Lange, W. (2013). Desigualdades educacionais em contextos urbanos: Um estudo da geografia de oportunidades educacionais na cidade do Rio de Janeiro. Educação É Sociedade, 34(125), 1175-1202. https://doi.org/10.1590/So101-73302013000400009

Koslinski, M. C., Costa, M. e Alves, F. (2014). Residential segregation, school quasi-market and school segmentation in the context of Rio de Janeiro. En L. Ribeiro (Org.), The metropolis of Rio de Janeiro: A space in transition (pp. 219-246). Routledge.

Marino, L. L. (2016). Análise de dados com característica longitudinal em educação: Um estudo sobre o município do Rio de Janeiro (Dissertação de Mestrado). Escola Nacional de Ciências Estatísticas IBGE.

Mendes, I. A. A. (2017). Território, segregação escolar e políticas de regulação de matrícula: Um estudo da cidade de Belo Horizonte (Tese Doutorado). Programa de Pós-Graduação em Educação. Universidade Federal de Minas Gerais.

Murillo, F. J. (2016). Midiendo la segregación escolar en América Latina. Un análisis metodológico utilizando el TERCE. REICE. Revista Iberoamericana sobre Calidad, Eficacia y Cambio en Educación, 14(4), 33-60. https://doi.org/10.15366/reice2016.14.4.002

Noreisch, K. (2007). Choice as rule, exception and coincidence: Parents' understandings of catchment areas in Berlin. Urban Studies, 44(7), 1307-1328.

https://doi.org/10.1080/00420980701302320

Quiroz, S. S., Dari, N. L. e Cervini, R. A. (2020). Oportunidad de aprender e segmentación socioeconómica en Argentina PISA 2015. REICE. Revista Iberoamericana sobre Calidad, Eficacia y Cambio en Educación, 18(3), 93-1 12. https://doi.org/10.15366/reice2020.18.3.005 
Rawls, J. (1971). A theory of justice. Harvard University Press.

Rosistolato, R., Pires do Prado, A. P., Muanis, M. C. e Cerdeira, D. G. (2019). Burocracia educacional em interação com as famílias nos processos de matrícula escolar na cidade do Rio de Janeiro. Jornal de Políticas Educacionais, 13, 1-28. https://doi.org/10.5380/jpe.v13io.68554

Santos, H. e Elacqua, G. (2016). Socioeconomic school segregation in Chile: Parental choice and theoretical counterfactual analysis. CEPAL Review, 119, 123-137. https://doi.org/10.18356/bf1 1809a-en

Saporito, S. (2003). Private choices, public consequences: Magnet school choice and segregation by race and poverty. Social Problems, 50(2), 181-203. https://doi.org/10.1525/sp.2003.50.2.181

Tymms, P. (1999). Baseline assessment and monitoring in primary schools: Achievements, attitudes and value-added indicators. David Fulton Publishers.

Tymms, P. (2004). Effect sizes in multilevel models. En I. Schagen e K. Elliot (Orgs.), But what does it mean? The use of effect sizes in educational research (pp. 79-112). National Foundation for Educational Research.

Van Zanten, A. (2005). Efeitos da concorrência sobre a atividade dos estabelecimentos escolares. Cadernos de Pesquisa, 35(126), 565-593.

https://doi.org/10.1590/So100-15742005000300003.

West, A., Hind, A. e Pennell, H. (2004). School admissions and "selection" in comprehensive schools: Policy and practice. Oxford Review of Education, 30(3), 347-369. https://doi.org/10.1080/0305498042000260485 


\section{Anexos}

Quadro A. Modelos de Regressão multinível - primeiro ano na pré-escola

\begin{tabular}{|c|c|c|c|c|c|c|}
\hline & \multicolumn{3}{|c|}{ LINGUAGEM } & \multicolumn{3}{|c|}{ MATEMÁTICA } \\
\hline & $\begin{array}{c}\text { Modelo } \\
1\end{array}$ & $\begin{array}{c}\text { Modelo } \\
\mathbf{2}\end{array}$ & $\begin{array}{c}\text { Modelo } \\
\mathbf{3}\end{array}$ & $\begin{array}{c}\text { Modelo } \\
1\end{array}$ & $\begin{array}{c}\text { Modelo } \\
\quad 2\end{array}$ & $\begin{array}{c}\text { Modelo } \\
\mathbf{3}\end{array}$ \\
\hline Intercepto & $0,253^{* * * *}$ & $0,345^{* * * *}$ & $0,269^{* * *}$ & $-1,875^{* * *}$ & $-1,821^{* * * *}$ & $-1,869^{* * * *}$ \\
\hline NSE (escola) & $0,083^{*}$ & $0,096^{* * * *}$ & $0,065^{*}$ & $0,126^{* * *}$ & $0,147^{* * * *}$ & $0,054^{+}$ \\
\hline Sexo & & $-0,127^{* * * *}$ & $-0,030$ & & $-0,026$ & 0,007 \\
\hline Idade & & $0,278^{* * * *}$ & $0,094^{* * * *}$ & & $0,454^{* * * *}$ & $0,138^{* * * *}$ \\
\hline SEN & & $-0.926^{* * * *}$ & $-0,405^{* * *}$ & & $-1, \mathrm{OOO}^{* * * *}$ & $-0,859^{* * * *}$ \\
\hline NSE (criança) & $0,169^{* * * *}$ & $0,176^{* * * *}$ & $0,061^{* * *}$ & $0,311^{* * * *}$ & $0,319^{* * * *}$ & $0,119^{* * * *}$ \\
\hline Matemática Onda 1 & & & & & & $0,937^{* * * *}$ \\
\hline Linguagem Onda 1 & & & $0,535^{* * *}$ & & & \\
\hline INTRCPT 1 , uO & 0,025 & 0,021 & 0,017 & 0,037 & 0,033 & 0,015 \\
\hline level-1, r & 0,653 & 0,566 & 0,338 & 1,670 & 1,488 & 0,784 \\
\hline $\mathrm{N} 1$ & 1955 & 1952 & 1951 & 1955 & 1952 & 1951 \\
\hline $\mathrm{N}_{2}$ & 46 & 46 & 46 & 46 & 46 & 46 \\
\hline
\end{tabular}

Nota: ${ }^{* * *} \mathrm{p}<0,001,{ }^{* *} \mathrm{p}<0,01,{ }^{*} \mathrm{p}<0,05,{ }^{+} \mathrm{p}<0,10$.

Fonte: Elaboração própria dos autores.

Quadro B. Modelos de Regressão multinível - segundo ano na pré-escola

\begin{tabular}{lcccccc}
\hline & \multicolumn{3}{c}{ LINGUAGEM } & \multicolumn{3}{c}{ MATEMÁTICA } \\
\cline { 2 - 7 } & Modelo & Modelo & Modelo & Modelo & Modelo & Modelo \\
& $\mathbf{1}$ & $\mathbf{2}$ & $\mathbf{3}$ & $\mathbf{1}$ & $\mathbf{2}$ & $\mathbf{3}$ \\
\hline Intercepto & $0,920^{* * *}$ & $1,038^{* * *}$ & $0,959^{* * *}$ & $-0,602^{* * *}$ & $-0,625^{* * *}$ & $-0,642^{* * *}$ \\
NSE (escola) & $0,132^{* * *}$ & $0,148^{* * *}$ & $0,069^{*}$ & $0,123^{* *}$ & $0,153^{* * *}$ & $0,055^{*}$ \\
Sexo & & $-0,150^{* * *}$ & $-0,056^{*}$ & & $-0,146^{*}$ & $0,193^{* * *}$ \\
Idade & & $0,247^{* * *}$ & $0,073^{* * *}$ & & $0,471^{* * * *}$ & $0,116^{* * * *}$ \\
SEN & & $-1,394^{* * *}$ & $-0,362^{* * *}$ & & $-1,850^{* * *}$ & $-0,903^{* * * *}$ \\
NSE (criança) & $0,171^{* * *}$ & $0,190^{* * *}$ & $0,051^{* * *}$ & $0,301^{* * *}$ & $0,321^{* * *}$ & 0,042 \\
Matemática Onda 2 & & & & & & $1,140^{* * *}$ \\
Linguagem Onda 2 & & & $0,672^{* * *}$ & & & \\
INTRCPT1, uO & 0,053 & 0,047 & 0,033 & 0,087 & 0,071 & 0,026 \\
\hline level-1, r & 0,819 & 0,708 & 0,334 & 2,224 & 1,938 & 0,836 \\
\hline N1 & 2704 & 2670 & 1920 & 2704 & 2670 & 1920 \\
\hline N2 & 46 & 46 & 46 & 46 & 46 & 46 \\
\hline Nota: & & & & &
\end{tabular}

Nota: ${ }^{* * *} \mathrm{p}<0,001,{ }^{* *} \mathrm{p}<0,01,{ }^{*} \mathrm{p}<0,05,{ }^{+} \mathrm{p}<0,10$.

Fonte: Elaboração própria dos autores.

\section{Breve CV dos autores}

\section{Tiago Lisboa Bartholo}

Professor do Programa de Pós-Graduação em Educação da Universidade Federal do Rio de Janeiro e do Colégio de Aplicação da Universidade Federal do Rio de Janeiro. Doutorado em Educação pela Universidade Federal do Rio de Janeiro e pesquisador do Laboratório de Pesquisas em Oportunidades Educacionais (LaPOpE/ UFRJ). Pesquisador visitante na Universidade de Durham, Inglaterra, (2017 e 2014), 
Universidade de Birmingham, Inglaterra, (2012 e 2013) e Universidade León, Espanha, (2009). Seus principais interesses de pesquisa são relacionados à temática das desigualdades educacionais, segregação escolar e avalição de impacto de programas e políticas educacionais. ORCID ID: https://orcid.org/0000-0002-2400-8707. Email: tiagobartholo@ufrj.com

\section{Mariane Campelo Koslinski}

Professora associada da Faculdade de Educação e do Programa de Pós-graduação em Educação da Universidade Federal do Rio de Janeiro. Doutorado em Sociologia pela Universidade Federal do Rio de Janeiro/UFRJ (2007) e Pós-Doutorado no Instituto de Pesquisa em Planejamento Urbano e Regional IPPUR/UFRJ (2008-2009). Pesquisadora visitante no Center of Organizational Innovation/Columbia University (2005-2006). Coordenadora do Laboratório de Pesquisa em Oportunidades Educacionais (LaPOpE/UFRJ). Coordenadora do GT 13 Educação e Sociedade da Sociedade Brasileira de Sociologia. Seus principais interesses de pesquisa são nas áreas de sociologia da educação (avaliação educacional), análise de políticas educacionais, desigualdades de oportunidades educacionais e segregação socioespacial e desigualdades educacionais. ORCID ID: https://orcid.org/O000-0002-9644-5041. Email: mckoslinski@ufrj.br

\section{Felipe Macedo de Andrade}

Professor do Colégio de Aplicação da Universidade Federal do Rio de Janeiro. Doutorado em Educação pela Universidade Federal do Rio de Janeiro e pesquisador do Laboratório de Pesquisa em Oportunidades Educacionais (LaPOpE/UFRJ). Coordenador do Curso de Especialização Saberes e Práticas na Educação Básica (CESPEB/ UFRJ) na ênfase de Educação Física Escolar. Seus principais interesses de pesquisa são sociologia da educação, políticas de responsabilização e avaliação em larga escala. ORCID ID: https://orcid.org/0000-0002-1153-2614. Email: felipema8@ufrj.br

\section{Daniel Lopes de Castro}

Graduado em Pedagogia pela Universidade Federal do Rio de Janeiro (2019) e Mestrando em Educação na linha de Políticas e Instituições Educacionais pelo Programa de PósGraduação em Educação da Universidade Federal do Rio de Janeiro. É bolsista CAPES do Programa de Excelência Acadêmica (PROEX) e pesquisador do Laboratório de Pesquisa em Oportunidades Educacionais (LaPOpE/ UFRJ). Atua em pesquisas de larga escala em Avaliação de Sistemas, Políticas Públicas e Programas Educacionais focados em Educação Infantil e anos iniciais do Ensino Fundamental. Atualmente estuda sociologia da educação com foco em desigualdades escolares na Educação Infantil e avaliação de larga escala. ORCID ID: https://orcid.org/o000-0001-8512-5520. Email: dcastro@ufrj.br 